\title{
Oral Cysticercosis: A Case Report and Review of Literature
}

\author{
Mahadevi B. Hosur · Sanjay Byakodi • \\ R. S. Puranik · Shreenivas S. Vanaki • \\ Surekha R. Puranik • M. S. Shivakumar
}

Received: 5 August 2014/ Accepted: 16 January 2015/Published online: 30 January 2015

(C) The Association of Oral and Maxillofacial Surgeons of India 2015

Keywords Cysticercosis - Diagnosis of cysticercosis . Oral cysticercosis · Parasiticinfection · Taenia solium

\section{Introduction}

Cysticercosis is a potentially fatal parasitic disease that rarely involves the oral region in humans [1]. Cysticercosis is the result of infection with the larval stage of the tapeworm, Taenia solium (T. solium) [2]. Cysticercus, the larval stage of $T$. solium, resides in muscle and other tissues in pigs that serve as intermediate host. Ingestion of inadequately cooked pork containing cysticerci leads to infection in human beings, the definitive host [2]. Human beings can also serve as intermediate hosts through accidental ingestion of $T$. solium eggs. Acquisition of cysticercosis is therefore mainly by the faecal-oral route and not necessarily by eating pork [2]. It usually affects subcutaneous tissue, brain, muscle, heart, liver, lungs and the eyes [3]. This is a more serious condition because the ingested eggs develop into embryos (oncospheres) that can penetrate the intestinal wall and disseminate through vascular or

M. B. Hosur $(\bowtie) \cdot$ R. S. Puranik · S. S. Vanaki .

M. S. Shivakumar

Department of Oral Pathology and Microbiology, PMNM Dental

College and Hospital, Bagalkot 587101, Karnataka, India

e-mail: maddy2006@rediffmail.com

S. Byakodi

Department of Oral and Maxillofacial Surgery, Bharati

Vidyapeeth Dental College and Hospital, Sangli, Maharashtra, India

S. R. Puranik

Department of Oral Medicine, Diagnosis and Radiology, PMNM

Dental College and Hospital, Bagalkot 587101, Karnataka, India lymphatic circulation to develop into cystic larvae (cysticercus cellulosae) [4].

Oral cysticercosis is a rare entity, is a diagnostic challenge and is poorly documented in the English language literature. Here we report a case of oral cysticercosis that emphasizes the importance of subjecting even apparently innocuous appearing lesions for histopathological evaluation and routine microscopic examination in diagnosing such lesions.

\section{Case Report}

An 18 year old male patient visited a private oral surgeon's clinic with the chief complaint of painless swelling in left cheek since 1 year. Patient was apparently alright 1 year back; then he noticed a small peanut sized soft swelling in the left inner cheek region which was slow growing. There was no history of trauma to the area, no history of discharge and no other swelling in the body. Patient gave a history of mixed dietary habit. His medical history was noncontributory. An intraoral examination revealed a well circumscribed, smooth submucosal, nodular swelling of approximately $1 \mathrm{~cm}$ in diameter in left buccal mucosa. Swelling was soft on palpation, non tender, non compressible and had an intact overlying mucosa (Fig. 1). Provisional diagnosis of lipoma was given by the clinician. Differential diagnosis of reactive lesions like focal fibrous hyperplasia, mucocele, benign mesenchymal tumor, and benign minor salivary gland tumor were considered. After obtaining the informed consent from the patient the clinician decided to go for surgical excision, under local anesthesia. Overlying mucosa was incised and lesion was found to be adherent to the underlying connective tissues and it was dissected out with sharp dissection and suturing 


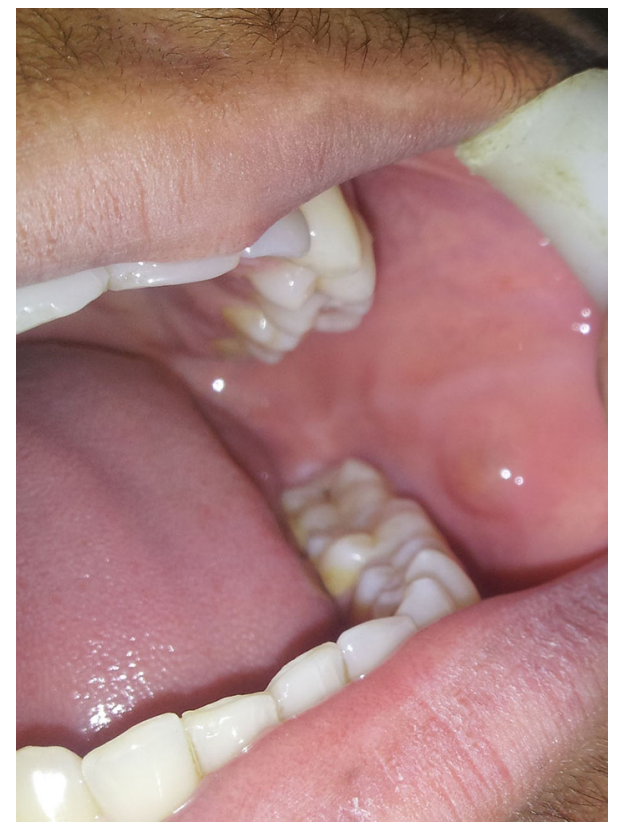

Fig. 1 Clinical photograph showing smooth, nodular swelling in left buccal mucosa

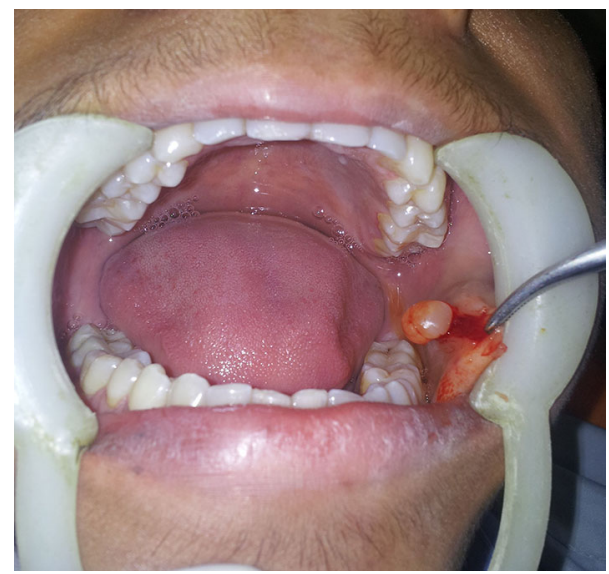

Fig. 2 Intraoperative photograph showing well defined, whitish transculecent cystic lesion

was done. Figure 2 shows the intraoperative picture of the soft cystic lesion. The tissue was sent to the department of Oral Pathology, PMNM Dental College and Hospital, Bagalkot, Karnataka for histopathological evaluation. On macroscopic examination lesion was well circumscribed, soft, and whitish in color (Fig. 3). Tissue was taken for processing. The Hematoxylin \& Eosin stained and studied sections showed cystic cavity covered by dense fibrous capsule (Fig. 4). The fibrous capsule comprised of a delicate double layered membrane consisting of an outer acellular hyaline eosinophilic layer and an inner cellular layer. Cyst consisted of duct like invaginations which were lined by eosinophilic membrane suggestive of parasites,

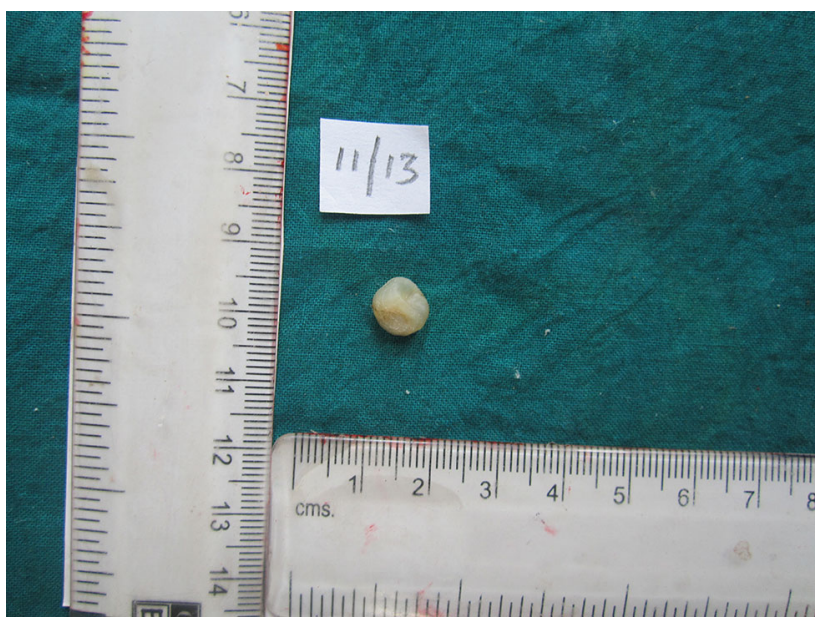

Fig. 3 Gross specimen photomicrograph

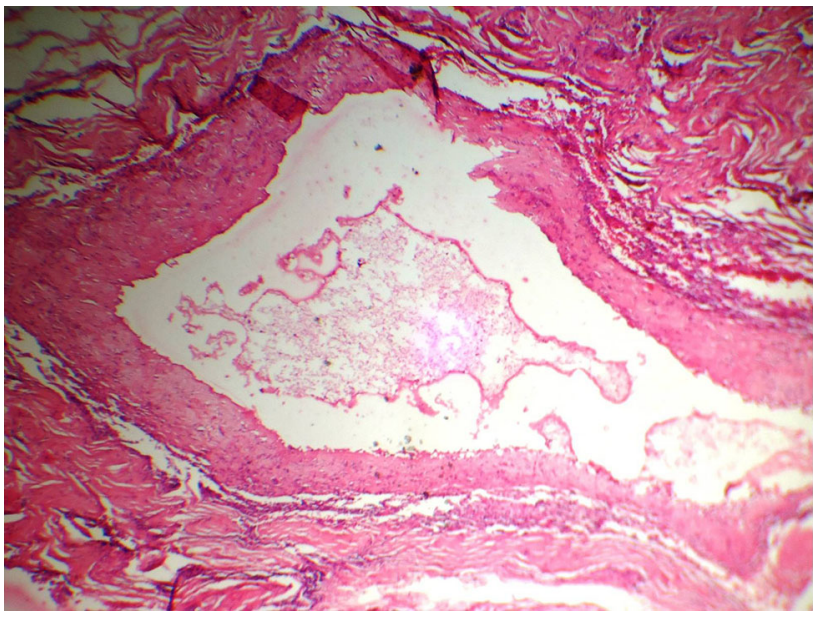

Fig. 4 Photomicrograph of low power view showing cystic lesion containing lumen lined by fibrous capsule

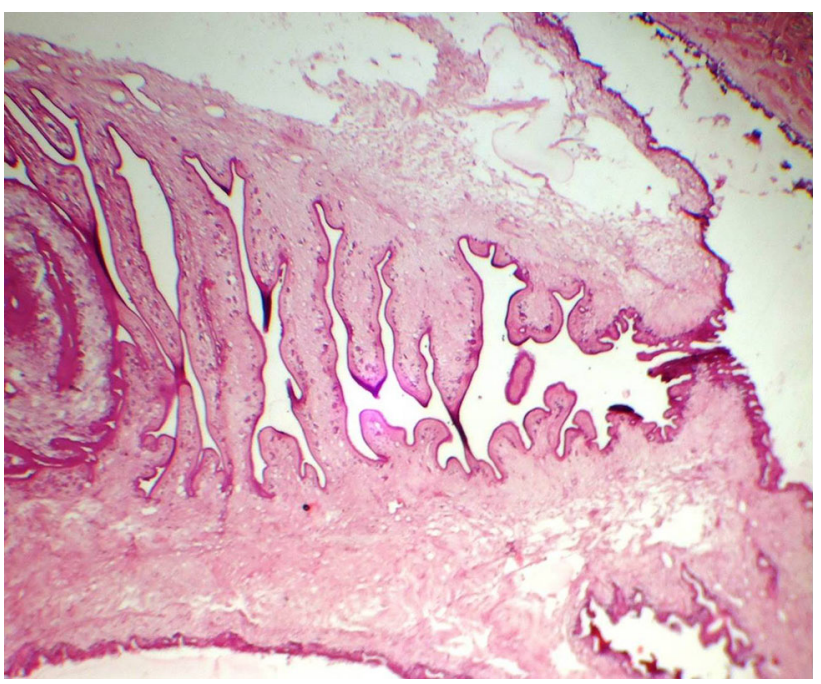

Fig. 5 Photomicrograph of the lesion shows dense fibrous capsule, inner aspect of the cyst shows duct like invaginations lined by double layer of eosinophilic membrane 
cysticercosis (Fig. 5). Higher magnification of the caudal region showed few ovoid basophilic calcified corpuscles. Diagnosis of oral cysticercosis was given based on histopathology.

\section{Discussion}

Cysticercosis is an infection with cysticercus cellulosae, the larval stage of Taenia solium or pork tape worm. $T$. solium passes its life cycle in two hosts. The definitive host is human who harbours the adult worm and an intermediate host is pig which harbours the larval stage. The adult worm lives in the small intestine of man. Usually one adult worm is present which lives for years. It is about three meters long with 1,000 proglotids (eggs). The worm sheds gravid segments laden with eggs in the stool which infect pigs [5]. In areas with poor oral hygiene the eggs (proglotids) excreted with faeces may be ingested by the pig, the intermediate host. The gastrointestinal secretions of the pig dissolve the eggs and liberate the oncospheres(embryos) in the intestinal wall and disseminate through the vascular and lymphatic circulations, so the larvae can be distributed to various tissues, especially muscle. When man consumes undercooked and contaminated pork, the larvae reach the intestine where they develop into adult tapeworms. This is the normal life cycle of $T$. solium [6]. Humans can also act as intermediate host of these worms. This happens by consumption of food, water, or through hands contaminated with eggs. The covering membrane of the eggs is digested by the gastric juice and the larvae are released into the intestine. Human cysticercosis results from the localization of the T. solium larvae in any site [6]. The larvae penetrate the mucosa, enter blood vessels and lymphatics and are distributed all over the body but preferentially locate to brain, muscle, skin, liver, lungs and heart. They are also found in the oral and perioral tissues, especially muscles of mastication. In tissues other than the intestinal mucosa, the larvae eventually die and are treated as foreign bodies, causing granuloma formation, scarring and calcification approximately 3 months later. These areas in the tissues are called as cysticerci [7].

Lustmann and Copelyn reported that Latin America, India, Eastern Europe, and Southern Africa are the endemic areas. However, the disease may also occur in non-endemic regions. In these countries cysticercosis is associated with increased immigration and travel-related intestinal tapeworm infection [6]. Cysticercosis has the predilection for striated muscles of neck, tongue, trunk, subcutaneous tissue, brain, liver, lungs, orbit, meninges, etc. [3].

Oral cysticercosis is a rare event. A thorough search in the PUBMED English literature reported 69 cases till date. Asymptomatic cystic swelling or nodule is the only evidence of the disease. Any region of the oral cavity may be involved but review of the reported cases suggests that tongue is a site of predilection with 32 of the 69 cases followed by labial and buccal mucosa [8]. When present in oral cavity it resembles a well circumscribed, soft, elastic, fluctuant swelling, often mimicking a mucocele [6]. Differential diagnosis depends on the location of the lesion [6]. Nodules on the lips and cheek may be considered as fibroma, lipoma, mucocele, pyogenic granuloma or pleomorphic adenoma. Nodules on the tongue may be considered as pyogenic granuloma, fibroma, granular cell tumour, or rhabdomyoma [1]. According to Wilson et al. oral cysticerci are firm nodules on palpation because of their intraluminal pressure and therefore neither lipoma nor hemangioma should be considered as clinical possibilities [1].

Although oral cysticercosis indicates disseminated infestation, systemic complications are not commonly detected in patients with oral lesions. This may be due to the fact that generally disseminated larvae are located in deep tissues and it may remain alive throughout the life of the host without giving clinical manifestations. Even so, patients with oral cysticercosis must be referred for medical evaluation [6]. Symptomatic disease usually results from invasion of the central nervous system, in particular when paranchymal neuro cysticerci die years after infection, resulting in strong inflammatory reaction that leads to epileptic seizures. Neurocysticercosis is the most common cause of acquired epilepsy in the developing world. Signs of raised intracranial pressure, motor deficits, and encephalitis are other manifestations of cerebral involvement. MRI of the brain is the investigation of choice in patients with suspected neurocysticercosis [2].

Neurocysticercosis (NCC) caused by the cysticerci of helminth $T$. solium has recently been associated with local malignant tumors particularly glioblastoma multiformae (GBM) and even neoplasia originating outside the nervous system e.g. the malignant haematological diseases. One study found NCC to be a risk factor for development of cerebral glioma. The possible mechanisms may be: (a) chronic inflammation leading to release of nitric oxide in brain which is a potential carcinogen. (b)The parasite induced modulation of host immune response leading to inhibition of tumour suppressor surveillance mechanisms. (c) The transfer of genetic material from parasite to host causes DNA damage thus predisposing to carcinogenesis [7]. In an epidemiological study, the NCC was found to be more frequent in cases with malignant haematological diseases than in controls. It has been shown that the chromosomal aberrations induced in peripheral lymphocytes during NCC could be an important factor. Further research is needed to confirm the potential role of cysticercosis in carcinogenesis [9]. 
The proposed relation between gliomas and NCC is complex. A direct association between gliomas and NCC has been reported and might be more than a coincidence. In a study by Del Brutto et al., it had been shown that NCC was more common among patients with cerebral gliomas than in controls. It has also been suggested that NCC may be the result and not the cause of the neoplasm, arguing that cancer-induced immunosuppression favours the development of parenchymal brain cysticerci. But this hypothesis seems unlikely, since NCC is not a common disease among patients immuno suppressed by other causes. The study by Del Brutto et al. also revealed that patients of glioma with NCC were older than those without NCC. It suggested a temporal relationship between NCC and the further development of a cerebral glioma and represented arguments against the hypothesis that NCC appeared as the result of cancer-induced immuno supression.

Some parasitic diseases have been implicated in the development of human cancers. It has been suggested that the inflammatory reaction induced by the parasites may cause some cells of the host to proliferate so much that they undergo mutations that alter their normal behaviour. Some cysticercal antigens stimulate the production of specific antibodies that form the basis for the immunological diagnosis of cysticercosis, while others (particularly antigen B) play a role in the evasion of the immune surveillance against cysticerci. In addition, it has been suggested that the occurrence of cellular immune dysfunction in patients with NCC, resulting from increased subpopulations of CD8 T-lymphocytes, impaired proliferation of lymphocytes, and abnormal concentration of cytokines. The depressed cellular immunity may be responsible for the reported association of NCC with conditions resulting from immunodeficiency states, and with the development of gliomas. In such cases, it has been hypothesized that the intense glial proliferation around the parasites, along with the suppression of the cellular immune responses may cause inhibition of the immunological surveillance against cancer, leading to malignant transformation of astrocytes. However, the association between NCC and gliomas was not reproduced in a more recent large study, and authors made the assumption that the coexistence of NCC with gliomas may be an incidental finding in patients from areas of high prevalence and endemicity [10].

Preoperative diagnosis of cysticercosis can be made by FNAC. Parts of the parasites have been identified in $45-100 \%$ of the aspirates. In the present case no FNAC was used as the lesion was thought to be representing some common lesions there. Other modes of diagnosis are examination of serum, saliva, cerebrospinal fluid analysis for antibodies to cysticercus cellulose by enzyme linked immunosorbent assay or enzyme linked immune electro transfer blot. Eosinophil count may be elevated or within normal limits [1].

Conventional radiographs are useful for the detection of calcification in muscles [9]. Relatively radiodense elongated images of the calcified cysticerci may appear on any radiograph of the soft tissues of the body [7]. In the head and neck region the locations of calcified cysticerci include muscles of mastication and facial expression, the suprahyoid muscle and the posterior cervical, as well as the tongue, buccal mucosa or lip. They are viewed as multiple well defined elliptical radiopacities resembling grains of rice [7]. Computed tomography (CT) and magnetic resonance imaging (MRI) have greatly improved the accuracy of diagnosis of cysticercosis, NCC in particular [10]. MRI is considered best for the detection of degenerating and viable cysticerci. The advent of high resolution ultrasound has greatly augmented the role of sinology in diagnosing muscular cysticercosis [10]. Nevertheless, the definitive diagnosis in all the previous published cases has been made by histopathology [1].

The treatment of oral cysticercosis is surgical enucleation. The anthelmintic drugs praziquantel and albendazole are effective in the treatment of disseminated cysticercosis [6]. Treatment in other locations is dependent on symptomatology and accessibility of the lesion to surgical intervention [1].

The prognosis in the maxillofacial region is good with no recurrences. In contrast to the gravity of the disease in the cerebral, ocular, or cardiac sites, it is often well-tolerated. When associated with other localizations the prognosis depends on site and number of larval localizations and may be severe [1].

Oral cysticerci, being localized and superficial lesions, are easy to excise and have good prognosis. Simple surgical excision is often all that is required to ensure complete removal of the lesion without any postoperative complications in such cases. However it is important to exclude the presence of parasites in other sites through detailed case study and systemic investigations [10]. In the present case though the lesion was excised which appears to suffice the treatment but no further investigations and follow up was possible for the possible involvement of other sites as patient had to leave to his native after excision and initially it was not suspected to be a case of cysticercosis on the clinical grounds.

The incidence of oral cysticercosis is rare and it should always be considered as a possibility when nodular formations appear in the mouth, especially when the affected individual lives or comes from a geographical area of high incidence of cysticercus. 


\section{Conclusion}

Oral cysticercosis should be considered in the differential diagnosis of intraoral solitary swellings of the oral cavity especially in endemic areas. The case reported here shows the importance of subjecting all the excised tissue specimens for histopathological evaluation, since neither the clinical examination nor a history suggested a diagnosis other than a common benign lesion to the clinician. So clinicians and oral pathologists should be aware of such uncommon lesion and should consider it in the differential diagnosis.

\section{References}

1. Deshmukh A, Avadhani A, Tupkari JV, Sardar M (2011) Cysticercosis of the upper lip. J Oral Maxillofac Pathol 15(2): 219-222

2. Jay A, Dhanda J, Chiodini PL, Woodrow CJ, Farthing PM, Evans $\mathbf{J}$ et al (2007) Oral cysticercosis. Br J Oral Maxillofac Surg 45(4):331-334
3. Elhence P, Bansal R, Sharma S, Bharat V (2012) Cysticercosis presenting as cervical lymphadenopathy: a rare presentation of two cases with review of literature. Niger $\mathbf{J}$ Clin Pract 15(3):361-363

4. Romero de Leon E, Aguirre A (1995) Oral cysticercosis. Oral Surg Oral Med Oral Pathol Oral Radiol Endod 79(5):572-577

5. Patel K, Shah M, Patel B, Doshi N (2011) Subcutaneous oral cysticercosis. Natl J Community Med 2:311-313

6. de Souza PE, Barreto DC, Fonseca LM, de Paula AM, Silva EC, Gomez RS (2000) Cysticercosis of the oral cavity: report of seven cases. Oral Dis 6(4):253-255

7. Cl Carter (2009) Soft tissue calcification and ossification. In: White SC, Pharoah MJ (eds) Oral Radiology Principals and Interpretation, 6th edn. Mosby, Philadelphia, p p529

8. Krishnamoorthy B, Suma GN, Dhillon M, Srivastava S, Sharma ML, Malik SS (2012) Encysted Tenia solium larva of the oral cavity: case report with review of literature. Contemp Clin Dent 3(suppl2):S228-S232

9. Khurana S, Dubey ML, Malla N (2005) Association of parasitic infections and cancers. Indian J Med Microbiol 23(2):74-79

10. Kumar N, Bhattacharya T, Kumar R, Radotra BD, Mukherjee KK, Kapoor R, Ghoshal S (2013) Is neurocysticercosis a risk factor for glioblastoma multiforme or a mere coincidence: a case report with review of literature. J Neurosci Rural Pract 4(1):67-69 